\title{
Engineering Issues for the Web 2.0
}

\author{
Gustavo Rossi • Qing Li • Yanchun Zhang
}

Published online: 10 September 2009

(C) Springer Science + Business Media, LLC 2009

\section{Editorial}

The Web has changed the way in which we work nowadays and it is not surprising that the Web itself is changing fast. From its inception as a static repository of documents in its youth as the site for different kinds of business and learning applications, it has become now a platform for multiple and disparate uses. New applications like social networks (in the style of Facebook, Hi5, etc.) and multimedia repositories (such as Flickr and Youtube) co-exist with the "old" news and e-commerce sites which have been improved with the ideas of this new generation of sites. At the same time the Web offers new interaction possibilities that facilitate desktop-like interfaces; it is also the way in which new software is built by mashing up distributed components using service-oriented communication. Creativity has been enhanced and every day we can discover new Webbased communities and blogs; millions of people contribute to Wikis and tag pages giving rise to a growing set of folksonomies. And with the advent of the Semantic Web we can give more meanings to the flat data in blogs, Wikis and social sites.

Some years ago the word Web 2.0 was coined to indicate the transition of the Web as a repository to the Web as a platform. Though many people argue that this name is just a buzzword because most of the so-called Web 2.0 applications were possible years before, and while the underlying Web technologies have not yet undergone a revolution, it is a matter of fact that more and more people associate the term Web 2.0 with the abovementioned applications and facilities.

Q. Li

Department of Computer Science, City University of Hong Kong, Hong Kong, China e-mail: itqli@cityu.edu.hk

G. Rossi (ه)

Lifia, F. Informatica, UNLP and Conicet, La Plata, Argentina

e-mail: gustavo@lifia.info.unlp.edu.ar

Y. Zhang

School of Engineering and Science, Victoria University, Melbourne, Australia

e-mail: Yanchun.Zhang@vu.edu.au 
Whatever the term we use to "tag" this new software generation, it is true that all these new applications have created new problems or, have re-shaped old problems. In this special issue we are interested in a broad set of problems, those related to the systematic construction of Web 2.0 applications and related tools. The articles in this issue cover a very wide range of problems from high-level design issues such as Design Patterns to lower-level ones such as algorithm optimization. They also span through different Web 2.0 applications, from social networks, to blogs. All papers have undergone a rigorous revision process. From the 25 submitted papers, we have selected six papers to be included in the special issue, which represents a first-hand vision in this enormous problem of engineering software for the Web 2.0.

In the first paper, "Web Engineering at the Frontier of the Web 2.0: Design Patterns for Online 3D Shared Spaces" by Nicoletta Di Blass, Franca Garzotto and Caterina Poggi, the authors argue that design experience, expressed in the form of Design Patterns is a key element to improve the construction of these social spaces; they distill their own experience in the development and evaluation of tri-dimensional shared spaces for youngsters.

The second paper, "Exploring the Relationship between Keywords and Feed Elements in Blog Post Search" by Seung-Kyun Han, Dongmin Shin, Jae-Yoon Jung and Jonghun Park, deals with the problem of searching blog posts. The authors argue that current blog search engines are not efficient for retrieving the most relevant posts for a user's query and present a ranking framework to improve search quality using relevance feedback and other important information for example from RSS feeds.

The third paper, "Social Propagation: Boosting Social Annotations for Web Mining" by Shenghua Bao, Bohai Yang, Ben Fei, Shengliang Xu, Zhong Su, and Yong Yu discusses the problem of boosting social annotations by using propagation. Social annotations are becoming increasingly popular and they help to improve web and enterprise search as well as Web browsing. However annotations are still sparse and cover only a small proportion of the Web. The authors propose a general propagation model to alleviate the problem of annotation sparseness.

The fourth paper, "The Effectiveness of Latent Semantic Analysis for Building Up a Bottom-up Taxonomy from Folksonomy Tags" by Takeharu Eda, Masatoshi Yoshikawa, Toshio Uchiyama, and Tadasu Uchiyama handles the problem of achieving more efficient use of folksonomy tags. With this aim the authors propose an organizing method based on Probabilistic Latent Semantic Indexing to discriminate subjective tags and building a hierarchy with the objective tags.

In the fifth paper "Reducing Query Latencies in Web Search using Fine-Grained Paralellism" by Eitan Frachtemberg, the author focuses on Semantic Web search and discusses the main challenges in the development of a semantic search engine that aims at answering queries by matching meaning in queries to meaning in Web Documents. He shows how to exploit data parallelism in slow search queries to reduce their latency in multicore systems.

Finally, in the sixth paper "Web-Traveler Policies for Images on Social Networks" by Anna C. Squicciarini and Smitha Sundareswaran, the authors analyze the problem of access control for images in social networks. The concept of Web-traveler policies is introduced to allow users to specify who can view, upload or download an image within the network. Such policies are innovative as they travel with the images while they are hosted in the social network.

\section{Table of contents}

- Web Engineering at the Frontier of the Web 2.0: Design Patterns for Online 3D Shared Spaces. Nicoletta Di Blass, Franca Garzotto and Caterina Poggi 
- Exploring the Relationship between Keywords and Feed Elements in Blog Post Search. Seung-Kyun Han, Dongmin Shin, Jae-Yoon Jung and Jonghun Park.

- Social Propagation: Boosting Social Annotations for Web Mining. Shenghua Bao, Bohai Yang, Ben Fei, Shengliang Xu, Zhong Su and Yong Yu

- The Effectiveness of Latent Semantic Analysis for Building Up a Bottom-up Taxonomy from Folksonomy Tags. Takeharu Eda, Masatoshi Yoshikawa, Toshio Uchiyama and Tadasu Uchiyama

- Reducing Query Latencies in Web Search using Fine-Grained Paralellism. Eitan Frachtemberg

- Web-Traveler Policies for Images on Social Networks. Anna C. Squicciarini and Smitha Sundareswaran 